\title{
Psicogeriatria e medicina generale
}

\author{
ALBERTO SPAGNOLI
}

Nelle liste dei medici di base le persone con più di 64 anni sono tra il 17 e il $33 \%$, gli ultra 75 enni vanno dall' 8 al $22 \%$. I risultati di una ricerca in corso, nell'ambito del progetto finalizzato Invecchiamento del CNR, mostrano che il $23 \%$ degli ultra 75 enni valutati a domicilio ha un deficit cognitivo mediograve, mentre il $26 \%$ delle donne e il $12 \%$ degli uomini presenta un quadro depressivo clinicamente rilevante. Da altri studi risulta che gli psicofarmaci e i cerebroattivi sono tra i farmaci più prescritti: la percentuale di anziani in terapia con queste sostanze è rispettivamente del 25 e del $26 \%$ (Spagnoli et al., 1989).

Demenza, depressione e psicofarmaci (compresi i cerebroattivi) costituiscono quindi i problemi centrali della psicogeriatria nell'ambito della medicina generale. Le cose principali da fare sono: migliorare la capacità diagnostica della demenza e della depressione grave; modificare l'uso degli psicofarmaci sulla base di criteri di razionalità più volte esposti ma largamente inapplicati; migliorare la gestione complessiva di questi malati, sperimentando varie modalità di interazione tra medico di base, specialista, altri operatori della sanità e dell'assistenza, e i relativi strumenti e procedure.

L'ampia letteratura sulla nuova nosografia delle patologie cognitive non è penetrata nella medicina di base e ciò comporta alcune conseguenze negative. Il medico, non differenziando i cali fisiologici di memoria, la depressione, le peculiarità del carattere, gli stati confusionali, le demenze - tutto questo può

Indirizzo per la corrispondenza: Dr. A. Spagnoli, Laboratorio di Neuropsichiatria Geriatrica, Istituto di Ricerche Farmacologiche Mario Negri, Via Eritrea 62, 20157 Milano.

Fax $(+39)$ 02-354.6277. infatti stare nell'arteriosclerosi cerebrale - non fa diagnosi delle forme demenziali potenzialmente (cioè: se diagnosticate e curate senza troppo ritardo) reversibili o delle gravi depressioni. L'imprecisione diagnostica è inoltre il presupposto per una terapia farmacologica scorretta. Infine, i parenti chiedono la diagnosi, una terapia, la prognosi, dei consigli sui problemi assistenziali e sulle pratiche di agevolazione economica da avviare, talora un giudizio sull'opportunità di un ricovero temporaneo o della definitiva istituzionalizzazione. Voglio dire che la diagnosi in psicogeriatria è importante, purché sia corretta e utilizzata in modo intelligente.

Qual è allora il posto giusto del medico di base nella gestione dei problemi psicogeriatrici? Va potenziata l'assistenza primaria o dobbiamo delegare la pianificazione e l'esecuzione degli interventi sanitari a gruppi di esperti (ad esempio, le Unità Valutative Geriatriche) (Hardy-Thompson et al., 1992)? Sono domande cruciali che, in assenza di un impegno culturale e di ricerca, ricevono risposte fondate su opzioni ideologiche $o$, più spesso, su logiche di potere. È chiaro comunque che la riaffermazione del ruolo del medico di medicina generale nella psicogeriatria - e nella geriatria - diventa velleitaria se non avviene nell'ambito di un sistema di assistenza primaria, che comprenda anche competenze riabilitative, infermieristiche e assistenziali. Ma la trasformazione è profonda, difficile, piena di problemi a mio parere largamente sottovalutati. È nota, ad esempio, l'importanza attribuita alle attività di valutazione e assistenza domiciliare, per le quali è comunque necessaria l'acquisizione di un orizzonte operativo che la grande maggioranza dei medici di base e degli infermieri non ha (Council on Scientific Affairs, 1991). Nessuno dà al medico le informazioni per capire il tipo di competenze e di tecniche impiegate da altri operatori della 
salute e dell'assistenza, e il tanto auspicato coordinamento dei servizi spesso si rivela un'illusione o il teatro di scontri tra tecnici che portano una visione diversa della salute e del rapporto tra sanità e assistenza (Clark, 1991). La scelta di credere nell'importanza - anche per la psicogeriatria - di una medicina di base rinnovata, deve quindi diventare argomento di ricerca.

I grandi obiettivi della psicogeriatria sono: curare le malattie curabili, alleviare i sintomi disturbanti e garantire assistenza in caso di malattia invalidante, prevenire la disabilità o ritardarne l'evoluzione. Il tutto nel rispetto della dignità e della libertà di scelta di malati e parenti, con un'ovvia opzione preferenziale per la permanenza a domicilio.

Attorno ai concetti di valutazione globale, autosufficienza, qualità della vita, sorgono nuove tecnologie e nuove proposte. È un momento fertile e felice per la psicogeriatria; lo è molto meno per gli anziani ammalati. Confesso di trovarmi a disagio con la qualità della vita; è un'espressione molto forte, il richiamo a una dimensione di ben-essere che trascende le possibilità della medicina (Callahan, 1990). La definizione di salute dell'OMS («...completo benessere fisico, psichico e sociale») ha certo liberato grandi energie, ma rischia ora di creare confusione, soprattutto in geriatria. Karl Jaspers (1991) dice: «Una salute di questo tipo non esiste. In base a questo concetto, in realtà, tutti gli uomini, in ogni istante, sono in qualche modo malati».

La comprensione del rapporto tra salute e invecchiamento può valersi con maggior profitto di tre concetti che definiscono altrettanti piani di discorso, tra loro interattivi. Il primo è il concetto di invecchiamento produttivo, derivato dall'economia e dalla sociologia; il secondo è quello di invecchiamento sano, cioè in assenza di malattie; il terzo è il concetto di invecchiamento compensato, che trova nella riabilitazione il referente teorico-pratico, e raffigura la situazione di persone malate ma funzionalmente e socialmente adattate (Curb, 1990). Per dare la dimensione epidemiologica di uno di questi costrutti, è utile ricordare che, durante lo studio del CNR condotto con i medici di base citato in precedenza, abbiamo verificato che negli ultra 75enni l'invecchiamento sano (completa autonomia nelle attività di base e strumentali, assenza di malattie, assenza di terapie farmacologiche continuative) riguarda il $5 \%$ dei maschi e il 2\% delle femmine. Questo modo di impostare il problema consente una via d'uscita dal riduzionismo del modello medico, pur conservandone gli innegabili vantaggi operativi.
Quanto detto vuole proporre il problema del limite, richiamare vincoli determinanti, pur se storicamente determinati. Lo sconfinamento oltre questi limiti è fastidioso soprattutto in geriatria, dove la tensione tra l'enfasi sulle res novae e la realtà dell'abbandono fa scintille. Ha ragione Goodwin (1991) quando ricorda che la giusta ribellione della gerontologia contro i pregiudizi negativi sull'invecchiamento rischia di portare alla mitizzazione della vecchiaia: invecchiare è bello; oppure: l'Alzheimer è una cosa, l'invecchiamento un'altra. In realtà $i$ dati epidemiologici e biologici disponibili non vanno certo, per ora, in questa direzione. Dice ancora Goodwin: «Questa nuova ideologia geriatrica [che rischia di rimuovere gli aspetti più negativi dell'invecchiamento dalla coscienza collettiva e dai programmi di ricercal ha una matrice profonda. Tutti noi stiamo invecchiando. È come se tutti gli oncologi avessero il cancro".

La situazione riscontra in una USL che opera in un'area metropolitana dell'Italia settentrionale - un luogo non certo svantaggiato rispetto al panorama nazionale (Anonimo, 1992) - può illustrare la distanza tra i bisogni e le risorse disonibili. Arrotondando un po' le cifre, la popolazione generale della USL è di 100000 abitanti; gli ultra 75enni - settore di popolazione a più alto rischio geriatrico e psicogeriatrico - sono 10000 e vivono quasi tutti $(97 \%)$ a domicilio. Per questa popolazione, che pur non esaurisce tutta l'area tradizionalmente riferibile agli anziani, sono validi i tassi di prevalenza della demenza e della depressione riportati in precedenza; in più, 1500 persone presentano una limitazione di varia gravità nell'esecuzione delle attività di base della vita quotidiana e 2500 in quelle strumentali. Circa 3400 anziani vivono soli. In quella stessa USL, gli assistenti sociali e domiciliari, per l'intera popolazione, sono rispettivamente 9 e 12; gli infermieri disponibili per visite domiciliari agli anziani sono 7 . Formazione specifica, attività di coordinamento, strumenti condivisi: zero. In simili condizioni è semplicemente ridicolo parlare di assistenza domiciliare.

Una considerazione finale, a proposito del rapporto tra idee, metodo di ricerca e pratica clinica. Sarà la filosofia della complessità, sarà perché dicono che le cose non hanno più un centro o perché abbiamo perso l'anima e acquistato tanti packages statistici, fatto sta che dietro al nitido cartello della epidemiologia descrittiva, arricchita dagli effetti speciali di una statistica sofisticata, abbondano studi ripetitivi, a volte davvero futili, che sarebbe più corretto definire di epidemiologia contemplativa, senza 
nulla togliere al fascino della contemplazione. Il problema è ancor più scottante in psicogeriatria date le condizioni disastrose della sanità e dell'assistenza. Dovremo quindi giungere ad un punto di equilibrio più avanzato tra studi descrittivi e studi di intervento, pensando a strategie di ricerca sul lungo periodo, che assumano la fase descrittiva come momento preliminare alla fase propriamente sperimentale - condotta su modelli assistenziali, terapie farmacologiche o riabilitative - , e al momento della trasformazione.

\section{BIBLIOGRAFIA}

Anonimo (1992). C'era una volta l'assistenza domiciliare. Regioni e politiche socio-assistenziali. Il Medico d'Italia, 7 marzo 1992.
Callahan D. (1990). What Kind of Life. The Limits of Medical Progress. Simon \& Schuster: New York.

Clark J. (1991). Nursing: as intellectual activity. British Medical Journal 303, 376-377.

Council on Scientific Affairs (1991). Educating physicians in home health care. Journal of American Medical Association 265, 769-771.

Curb J. D., Guralnik J. M., La Croix A. Z., Korper S. P., Deeg D., Miles T. \& White L. (1990). Effective aging. Meeting the challenge of growing older. Journal of American Geriatrics Society 38, 827-828.

Goodwin J. S. (1991). Geriatric ideology: the myth of the myth of senility. Journal of American Geriatrics Society 39, 627-631.

Hardy-Thompson C., Orrel M. W. \& Bergmann K. (1992). Evaluating a psychogeriatric domicilary visit service: views of general practitioners. British Medical Journal 304, 421-422.

Jaspers K. (1991). Il Medico nell'Età della Tecnica. Cortina: Milano. Spagnoli A., Ostino G., Dana Borga A., D'Ambrosio R., Maggiorotti P., Todisco E., Prattichizzo W., Pia L. \& Comelli M. (1989). Drug compliance and unreported drugs in the elderly. Journal of American Geriatrics Society 37, 619-624. 


\section{A. Scott Henderson}

\section{Psichiatria sociale ed epidemiologia psichiatrica}

Edizione italiana a cura di Michele Tansella

Un libro di 226 pagine. Lire 40.000

"L'opera di Scott Henderson rappresenta innanzitutto una fonte preziosa di informazioni e di dati, essendo l'autore riuscito a concentrare in nove capitoli i risultati dei principali studi di epidemiologia psichiatrica pubblicati finora.

Ma questo volume ha anche un altro pregio. Esso ricorda continuamente al lettore l'importanza dell'approceio epidemiologico e di un'adeguata metodologia di ricerca per testare in modo corretto le nostre ipotesi".

Dalla Presentazione del Curatore dell'edizione italiana

\section{Il Pensiero Scientifico Editore}

via Bradano 3/c - 00199 Roma

(06) 86207158 - 86207159 - 86207168 - 86207169

fax (06) 86207160

Compilate e spedite in busta chiusa a:

In Pensiero Scientifico Editore

$\square$ Sĩ DESIDERO RICEVERE N ${ }^{\circ}$ di "PSICHIATRIA SOCIALE"

al prezzo di Lire 40.000 la copia

VERSERO LA SOMMA DI LIRE

$\square$ VERSAMENTO C/C POSTALE $N^{\circ} 902015$

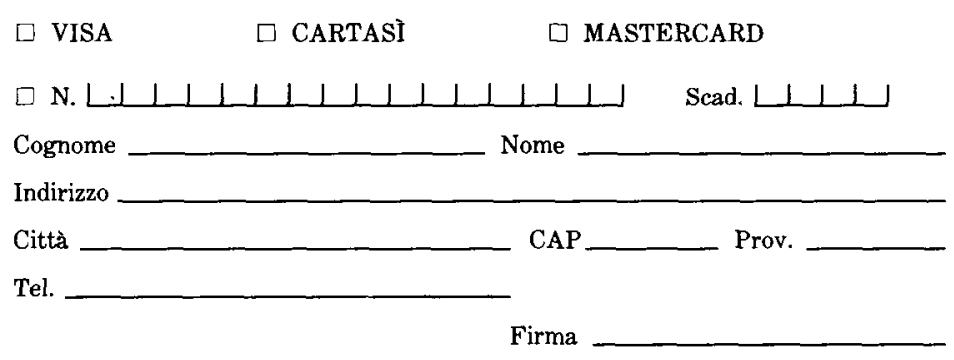

